\title{
Recours au télédépistage pour la rétinopathie diabétique
}

\author{
Daniel Rosenberg BSc, Jason Noble MD, Varun Chaudhary MD
}

Citation : CMAJ 2021 July 5;193:E1006. doi : 10.1503/cmaj.202141-f

Voir la version anglaise de l'article ici : www.cmaj.ca/lookup/doi/10.1503/cmaj.202141

\section{Un examen des yeux est essentiel pour détecter la rétinopathie diabétique}

Diabète Canada recommande un examen de la vue tous les 1-2 ans après un diagnostic de diabète de type $2^{1}$. Une revue systématique a montré qu'en moyenne 175 examens (75-267) sont nécessaires pour détecter un cas de rétinopathie diabétique pouvant causer une perte de vision chez les patients sans antécédent de rétinopathie ${ }^{2}$.

\section{Le télédépistage est un complément utile à l'examen en personne}

Le télédépistage de la rétinopathie consiste en la transmission d'images oculaires numériques obtenues par un technicien aux fins d'évaluation à distance par un spécialiste (annexe 1, accessible en anglais au www.cmaj.ca/lookup/ doi/10.1503/cmaj.202141/tab-related-content). Les techniciens doivent généralement suivre une formation individuelle avec un expert en téléophtalmologie pour saisir efficacement les images ${ }^{3}$. Des données probantes de grande qualité montrent une sensibilité et une spécificité supérieures à $95 \%$ dans le télédépistage de la rétinopathie diabétique ${ }^{1}$. Ce mode de dépistage est également moins coûteux que l'examen en personne ${ }^{3}$.

Les patients diabétiques sont plus susceptibles de se soumettre au télédépistage qu'à un examen en personne

Une récente méta-analyse a montré que le taux d'assiduité des patients était beaucoup plus élevé pour le télédépistage que pour l'examen traditionnel en personne (rapport de cotes : 13,15, intervalle de confiance à $95 \%, 8,01-21,61$, $p<0,001)^{4}$. Les patients en mauvaise santé ayant un accès limité aux professionnels de la santé ont de la difficulté à se présenter à l'examen en personne. Ils constituent une population cible pour le télédépistage de la rétinopathie ${ }^{1}$.

Les lignes directrices fondées sur des données probantes pour le télédépistage de la rétinopathie ne s'appliquent qu'aux patients de 12 ans et plus atteints du diabète de type 2 (ne s'appliquent pas aux femmes enceintes) ${ }^{5}$. II arrive que les images obtenues au cours du télédépistage soient jugées « non évaluables » et qu'un examen en personne soit nécessaire ${ }^{1}$. Les problèmes de santé qui peuvent interférer avec l'acquisition d'images numériques incluent la cataracte dense et les taies cornéennes ${ }^{6}$.

\section{Des systèmes centralisés d'orientation vers le télédépistage sont en train d'être mis en place au Canada}

Plusieurs cliniques de télédépistage de la rétinopathie diabétique ont été créées au Canada ${ }^{1}$. Les fournisseurs de soins primaires sont invités à communiquer avec la Société canadienne de la rétine (www.crssrc.ca) pour obtenir de plus amples renseignements sur l'orientation vers les programmes de télédépistage.

\section{Références}

1. Hooper P, Boucher M, Cruess A, et al. Excerpt from the Canadian Ophthalmological Society evidence-based clinical practice guidelines for the management of diabetic retinopathy. Can J Ophthalmol 2017;52(Suppl 1): S45-74.

2. Groeneveld Y, Tavenier D, Blom JW, et al. Incidence of sight-threatening diabetic retinopathy in people with type 2 diabetes mellitus and numbers needed to screen: a systematic review. Diabet Med 2019;36:1199-208.

3. Kim J, Driver DD. Teleophthalmology for first nations clients at risk of diabetic retinopathy: a mixed methods evaluation. JMIR Med Inform 2015;3:e10.

4. Kawaguchi A, Sharafeldin N, Sundaram A, et al. Teleophthalmology for age-related macular degeneration and diabetic retinopathy screening: a systematic review and meta-analysis. Telemed J E Health 2018;24: 301-8.

5. Boucher MC, Qian J, Brent MH, et al.; Steering Committee for Tele-Ophthalmology Screening, Canadian Retina Research Network. Evidence-based Canadian guidelines for tele-retina screening for diabetic retinopathy: recommendations from the Canadian Retina Research Network (CR2N) Tele-Retina Steering Committee. Can J Ophthalmol 2020;55(Suppl 1):14-24.

6. Silva PS, Horton MB, Clary D, et al. Identification of diabetic retinopathy and ungradable image rate with ultrawide field imaging in a national teleophthalmology program. Ophthalmology 2016;123:1360-7. 
Intérêts concurrents : Jason Noble siège au conseil consultatif de Novartis et de Bayer et est conférencier pour Novartis. Varun Chaudhary a siégé aux comités consultatifs de Novartis, de Bayer et de Roche et a été consultant pour ces entreprises. II touche des subventions de recherche parrainée par des chercheurs et participe à des essais cliniques financés par Novartis, Bayer et Allergan. Tous ces intérêts concurrents sont indépendants des travaux soumis. Aucun autre intérêt concurrent n'a été déclaré.

Cet article a été révisé par des pairs.

Affiliations : École de médecine Michael G. DeGroote (Rosenberg), Université McMaster, Hamilton, Ont.; Département d'ophtalmologie et des sciences de la vision (Noble); Centre des sciences de la santé Sunnybrook (Noble), Toronto, Ont.; Département de chirurgie, Division d'ophtalmologie (Chaudhary); Département des méthodes, des données et de l'incidence de la recherche en santé (Chaudhary), Université McMaster; Centre de soins de santé St. Joseph's de Hamilton (Chaudhary), Hamilton, Ont.

Propriété intellectuelle du contenu : Il s'agit d'un article en libre accès distribué conformément aux modalités de la licence Creative Commons Attribution (CC BY-NCND 4.0), qui permet l'utilisation, la diffusion et la reproduction dans tout médium à la condition que la publication originale soit adéquatement citée, que l'utilisation se fasse à des fins non commerciales (c.-à-d., recherche ou éducation) et qu'aucune modification ni adaptation n'y soit apportée. Voir : https://creativecommons.org/ licenses/by-nc-nd/4.0/deed.fr.

Correspondance: Varun Chaudhary, vchaudh@mcmaster.ca

Le CMAJ vous invite à soumettre vos textes pour la rubrique "Cinq choses à savoir ... » en ligne à http://mc.manuscriptcentral.com/cmaj. 